\title{
Traveling Wave Solutions and Infinite-Dimensional Linear Spaces of Multiwave Solutions to Jimbo-Miwa Equation
}

\author{
Lijun Zhang ${ }^{1,2}$ and C. M. Khalique ${ }^{2}$ \\ ${ }^{1}$ Department of Mathematics, School of Science, Zhejiang Sci-Tech University, Hangzhou, Zhejiang 310018, China \\ ${ }^{2}$ Department of Mathematical Sciences, International Institute for Symmetry Analysis and Mathematical Modelling, \\ North-West University, Mafikeng Campus, Private Bag X 2046, Mmabatho 2735, South Africa
}

Correspondence should be addressed to Lijun Zhang; li-jun0608@163.com

Received 23 January 2014; Accepted 19 March 2014; Published 9 April 2014

Academic Editor: Weiguo Rui

Copyright (C) 2014 L. Zhang and C. M. Khalique. This is an open access article distributed under the Creative Commons Attribution License, which permits unrestricted use, distribution, and reproduction in any medium, provided the original work is properly cited.

\begin{abstract}
The traveling wave solutions and multiwave solutions to $(3+1)$-dimensional Jimbo-Miwa equation are investigated in this paper. As a result, besides the exact bounded solitary wave solutions, we obtain the existence of two families of bounded periodic traveling wave solutions and their implicit formulas by analysis of phase portrait of the corresponding traveling wave system. We derive the exact 2 -wave solutions and two families of arbitrary finite $N$-wave solutions by studying the linear space of its Hirota bilinear equation, which confirms that the $(3+1)$-dimensional Jimbo-Miwa equation admits multiwave solutions of any order and is completely integrable.
\end{abstract}

\section{Introduction}

Various nonlinear partial differential equations (NLPDEs) have been proposed to model different kinds of phenomena in natural and applied sciences such as fluid dynamics, plasma physics, solid-state physics, optical fibers, acoustics, mechanics, biology, and mathematical finance. Obviously, it is of significant importance to study the solutions of such NLPDEs from both theoretical and practical points of view. However, the solution spaces of nonlinear equations are infinite-dimensional and contain diverse solution structures, so it is usually a difficult job to determine the solutions to nonlinear NLPDEs.

A great idea to generate exact solutions of NLPDEs is to reduce the NLPDEs into some algebraic equations by assuming the solutions to have some special forms or satisfy some solvable simpler equations. This can be seen in, for example, the exp-function method [1], the tanh function method [2], the homogeneous balance method $[3,4]$, the auxiliary function method $[5,6]$, the sech-function method [7], the sine-cosine method $[8,9]$, the tanh-coth method [10], the Jacobi elliptic function method [11], the $F$-expansion method, and the extended $F$-expansion method [12]. Normally, it is not an easy task to solve these nonlinear algebraic equations reduced from NLPDEs because they involve many parameters. However, the rapid development of symbolic computation makes it relatively easy to solve these algebraic equations [13]. At the same time one should be very careful when applying these methods as different methods might give the same solutions. For example, solutions obtained by using the sech-function method, the tanh-coth method, and the exp-function method are actually the same $[14,15]$, because $f\left(\operatorname{sech}^{2} x\right)=f\left(1-\tanh ^{2} x\right)=f\left(4 /\left(e^{x}+e^{-x}\right)^{2}\right)$, for any function $f$.

Recently, the planar dynamical system theorem has been employed to study the traveling wave solutions of NLPDEs $[16,17]$. The best advantage of this approach is that the boundedness, periodicity, the shapes of the solutions, and even the singular traveling wave solutions of NLPDEs can be recognized easily from the corresponding orbits of their phase portraits under various different parametric conditions. Also the exact solutions can be derived at the same time. It is worth pointing out that Hirota's bilinear method [18] is also an amazing method to find exact solutions to some NLPDEs. By some independent variable transformation, various nonlinear equations of mathematical physics are transformed into Hirota's bilinear equations $[18,19]$, which possess some specific properties and thus might be applied 
to study the solution sets of nonlinear differential equations. Recently, even some programs have been designed and some algorithms have been proposed on searching for integrable bilinear equations [20-26]. Based on Hirota's bilinear form, soliton solutions were obtained by the Hirota perturbation technique [18], the multiple exp-function algorithm [27], and other methods [28-31]. Even some generalized bilinear form has been proposed recently by $\mathrm{Ma}$ [32].

The $(3+1)$-dimensional Jimbo-Miwa equation

$$
u_{x x x y}+3\left(u_{x} u_{y}\right)_{x}+2 u_{y t}-3 u_{x z}=0
$$

was first proposed and studied by Jimbo and Miwa [33]. Recently, this equation has attracted a great deal of attention which mainly focuses on its solutions, integrability properties and symmetries. Ma and Lee [34] proposed a direct approach to solve (1) by using rational function transformations. Li and Dai [35] applied the generalized Riccati equation method to look for its exact solutions. However, in [36], Kudryashov and Sinelshchikov pointed out some mistakes and proved that some solutions in [35] could be written in a uniform form and thus they are not new at all.

The $(3+1)$-dimensional Jimbo-Miwa equation can be transformed into the Hirota bilinear equation

$$
\left(D_{x}^{3} D_{y}+2 D_{t} D_{y}-3 D_{x} D_{z}\right) f \cdot f=0
$$

through the dependent variable transformation $u=2(\ln f)_{x}$.

In this paper, we firstly study the bounded traveling wave solutions of the Jimbo-Miwa equation (1) by investigating the bifurcation and phase portraits of a planar cubic polynomial ordinary differential equation by using the planar dynamical system theory $[16,37]$. We then explore the 1-wave and 2wave solutions and the infinite-dimensional linear spaces of multiwave solutions to Jimbo-Miwa equation by employing Hirota's bilinear method, thus confirming that Jimbo-Miwa equation possesses multiwave solutions of arbitrary order.

\section{Traveling Wave Solutions to the $(3+1)$-Dimensional Jimbo-Miwa Equation}

To investigate the traveling wave solutions to the $(3+$ 1)-dimensional Jimbo-Miwa equation, we make the traveling wave transformation $u(x, y, z, t)=u(\xi), \xi=k x+l y+$ $m z+n t$, under which (1) is reduced to the nonlinear ordinary differential equation

$$
k^{3} l \frac{d^{4} u}{d \xi^{4}}+6 k^{2} l \frac{d u}{d \xi} \frac{d^{2} u}{d \xi^{2}}+(2 \ln -3 k m) \frac{d^{2} u}{d \xi^{2}}=0 .
$$

Integrating (1) once with respect to $\xi$ gives

$$
k^{3} l \frac{d^{3} u}{d \xi^{3}}+3 k^{2} l\left(\frac{d u}{d \xi}\right)^{2}+(2 \ln -3 k m) \frac{d u}{d \xi}=g,
$$

where $g$ is an arbitrary constant. Let $v=d u / d \xi$; then (1) becomes

$$
k^{3} l \frac{d^{2} v}{d \xi^{2}}+3 k^{2} l v^{2}+(2 \ln -3 k m) v=g
$$

which is a second-order nonlinear ordinary differential equation. We will study the solutions of (5) by planar dynamical system method and thus derive the traveling wave solutions to the $(3+1)$-dimensional Jimbo-Miwa equation.

2.1. Bounded Solutions of (5). First, we rewrite (5) in a simpler and more general form, namely,

$$
\frac{d^{2} v}{d \xi^{2}}=a v^{2}+b v+c
$$

where $a=-3 / k, b=(3 k m-2 \ln ) /\left(k^{3} l\right)$, and $c=g /\left(k^{3} l\right)$. We now study the bifurcation and exact solutions of (6). Let $v^{\prime}=w$; then (6) is equivalent to the dynamical system

$$
\begin{gathered}
v^{\prime}=w, \\
w^{\prime}=a v^{2}+b v+c,
\end{gathered}
$$

which has the Hamiltonian

$$
H(v, w)=\frac{w^{2}}{2}-\frac{a}{3} v^{3}-\frac{b}{2} v^{2}-c v .
$$

Clearly, the phase orbits defined by the vector fields of system (7) determine all solutions of (6). The bounded solutions of (6) correspond to the bounded phase orbits of system (7), which we now investigate. Along the orbit corresponding to $H(v, w)=h$,

$$
\left(\frac{d v}{d \xi}\right)^{2}=\frac{2 a}{3} v^{3}+b v^{2}+2 c v+2 h .
$$

Consequently, the general formula of the solutions of (6) can be expressed as

$$
\int_{0}^{v} \frac{d v}{\sqrt{\left(2 a v^{3} / 3\right)+b v^{2}+2 c v+2 h}}= \pm \int_{\xi_{0}}^{\xi} d \xi .
$$

However, it is not easy to know the properties and the shapes of (10) which actually is determined by the parameters $a, b, c$, and $h$. Clearly, the abscissas of equilibrium points of system (7) are the zeros of $a v^{2}+b v+c=0$. Obviously, the system has no bounded orbits when $b^{2}-4 a c<0$. We suppose that $b^{2}-4 a c>0$ in order to study the bounded orbits of system (7). Denoting $v_{ \pm}=\left(-b \pm \sqrt{b^{2}-4 a c}\right) / 2 a$, then $E_{+}\left(v_{+}, 0\right)$ and $E_{-}\left(v_{-}, 0\right)$ are two equilibrium points of system (7). By the theory of planar dynamical system, we know that $E_{+}$is a saddle point and $E_{-}$is a center. Denote $h_{ \pm}=H\left(v_{ \pm}, 0\right)$, and, by careful computation, we get

$$
h_{ \pm}=\frac{\left(b^{2}-4 a c\right)\left(-b \pm \sqrt{b^{2}-4 a c}\right)+2 a b c}{12 a^{2}} .
$$

Obviously, $h_{-}<h<h_{+} \cdot H(v, w)=h_{+}$corresponds to homoclinic orbits, and $H(v, w)=h_{-}$corresponds to the center $E_{-}$and $H(v, w)=h$, where $h_{+}<h<h_{-}$corresponds to a family of closed orbits surrounding the center $E_{-}$, which are surrounded by a homoclinic orbit. That is to say, (10) defines 
bounded solutions if and only if $h_{+} \leq h<h_{-}$. To be exact, (10) defines a family of periodic solutions when $h_{+}<h<h_{-}$.

When $h=h_{+}$, (10) defines a bounded solution which approaches $v_{+}$as $\xi$ goes to infinity. Actually,

$$
\frac{2 a}{3} v^{3}+b v^{2}+2 c v+2 h_{+}=\frac{2 a}{3}\left(v-v_{+}\right)^{2}\left(v-v_{0}\right),
$$

where $v_{0}=-\left(b+2 \sqrt{b^{2}-4 a c}\right) / 2 a$, so (10) can be reduced to

$$
\int_{v_{0}}^{v} \frac{d v}{\left(v-v_{+}\right) \sqrt{a\left(v-v_{0}\right)}}=\sqrt{\frac{2}{3}}\left(\xi-\xi_{0}\right),
$$

from which we can get the exact solution as

$$
v=v_{+}-\left(v_{+}-v_{0}\right) \operatorname{sech}^{2}\left(\sqrt{\frac{a\left(v_{+}-v_{0}\right)}{6}}\left(\xi-\xi_{0}\right)\right) \text {. }
$$

By further simplification, (14) becomes

$$
v=v_{+}-\frac{3 \sqrt{b^{2}-4 a c}}{2 a} \operatorname{sech}^{2}\left[\frac{1}{2}\left(b^{2}-4 a c\right)^{1 / 4}\left(\xi-\xi_{0}\right)\right],
$$

which is an exact bounded solution of (5).

Thus, we have the following lemma.

Lemma 1. The general second-order ODE (6) has bounded solutions if and only if $b^{2}-4 a c>0$. The bounded solutions can be expressed as (10) in an implicit form. In fact, provided $h_{-}<h<h_{+},(10)$ defines a family of bounded periodic solutions and $h=h_{+}$defines a bounded solution which approaches $v_{+}$as $\xi$ goes to infinity and can be expressed explicitly as (15), where $v_{+}=\left(-b+\sqrt{b^{2}-4 a c}\right) / 2 a$ and $h_{ \pm}$is defined by (11).

2.2. Bounded Traveling Wave Solutions to the $(3+1)$-Dimensional Jimbo-Miwa Equation. According to the analysis and results in Section 2.1, we know that (5) has only two kinds of bounded solutions, among which one is a family of periodic solutions and another is a family of solutions approaching a fixed number as $\xi$ goes to infinity. Note that what we aim to study is the bounded traveling wave solutions to the $(3+1)$ dimensional Jimbo-Miwa equation which are determined by $v=d u / d \xi$ and $v$ satisfies (5). So we have to investigate how we can get the bounded solutions to (3) from the bounded solution of (5).

Clearly, $u(\xi)=\int_{\xi_{0}}^{\xi} v(\xi) d \xi$, and $v(\xi)$ can be expressed implicitly as (10). By the geometry meaning of the integral and the properties of the solutions of (5), we get the traveling wave solutions to the $(3+1)$-dimensional Jimbo-Miwa equation. To get the bounded solution, we choose the integral constant $g$ to be 0 ; that is, $c=0$ in (15), and so

$$
u(\xi)=g-\frac{3 \sqrt{|b|}}{a} \tanh \left[\frac{\sqrt{|b|}}{2}\left(\xi-\xi_{0}\right)\right] ;
$$

that is,

$$
\begin{aligned}
u(\xi)= & g-k \sqrt{\left|\frac{2 \ln -3 k m}{k^{3} l}\right|} \\
& \times \tanh \left[\frac{1}{2} \sqrt{\left|\frac{2 \ln -3 k m}{k^{3} l}\right|}\left(\xi-\xi_{0}\right)\right],
\end{aligned}
$$

which is a family of exact bounded kink traveling wave solutions to the $(3+1)$-dimensional Jimbo-Miwa equation, where $g$ is an arbitrary constant.

However, we may not get bounded solutions from the family of periodic solutions of (5). It is easy to see that if $v(\xi)$ is a periodic solution of (5), then $u(\xi)=\int_{\xi_{0}}^{\xi} v(\xi) d \xi$ is bounded if and only if $\int_{0}^{T} v(\xi) d \xi=0$, where $T$ is the period of the function $v(\xi)$. Recall that the period of the function $v(\xi)$ which is defined by (10) with $h_{-}<h<h_{+}$continuously depends on the parameters $a, b, c$, and $h$. So $\int_{0}^{T} v(\xi) d \xi$ continuously depends on the parameters $a, b, c$, and $h$ too. Let $V(a, b, c, h)=\int_{0}^{T} v(\xi) d \xi$; then $V(a, b, c, h)$ is a continuous function of $a, b, c$, and $h$. We now prove the existence of the root of $V(a, b, c, h)=0$ to get the existence of the bounded periodic traveling wave solutions to the $(3+1)$-dimensional Jimbo-Miwa equation. $h_{+}$,

By the theory of planar dynamical system, when $h_{-}<h<$

$$
\frac{2 a}{3} v^{3}+b v^{2}+2 c v+2 h_{+}=\frac{2 a}{3}\left(v-v_{1}\right)\left(v-v_{2}\right)\left(v-v_{3}\right),
$$

where $v_{1}>v_{+}>v_{2}>v_{-}>v_{3}>v_{0}$ in the case of $a>0$ and $v_{0}>v_{1}>v_{-}>v_{2}>v_{+}>v_{3}$ in the case of $a<0$. The periodic solution satisfies $v_{+}>v_{2}>v(\xi)>v_{3}>v_{0}$ when $a>0$ and $v_{0}>v_{1}>v(\xi)>v_{2}>v_{+}$when $a<0$. Clearly, $v_{0}>0$ when $a>0$ and $0>b>-4 \sqrt{a c / 3}$. So $v(\xi)>0$ and thus $V(a, b, c, h)>0$ when $a>0$ and $0>b>-4 \sqrt{a c / 3}$. However, $v(\xi)<v_{+}<0$ and thus $V(a, b, c, h)<0$ when $a>0, c>0$, and $b>0$. There must exist at least one zero of $V(a, b, c, h)=0$ in the region $a>0$ of the parameter space since $V(a, b, c, h)$ is a continuous function of $a, b, c$, and $h$. The same happens in the region $a<0$ of the parameter space. So, we know that there exist at least two families of bounded periodic traveling wave solutions to the $(3+1)$-dimensional Jimbo-Miwa equation. Thus we have the following theorem.

Theorem 2. The $(3+1)$-dimensional Jimbo-Miwa equation has two types of bounded traveling wave solutions as given below.

(1) The $(3+1)$-dimensional Jimbo-Miwa equation has a family of exact bounded kink traveling wave solutions

$$
\begin{aligned}
& u(x, y, z, t) \\
& =g-k \sqrt{\left|\frac{2 \ln -3 k m}{k^{3} l}\right|} \\
& \quad \times \tanh \left[\frac{1}{2} \sqrt{\left|\frac{2 \ln -3 k m}{k^{3} l}\right|}\left(k x+l y+m z+n t-\xi_{0}\right)\right],
\end{aligned}
$$

where $\xi_{0}$ and $g$ are two arbitrary constants. 
(2) The $(3+1)$-dimensional Jimbo-Miwa equation has at least two families of bounded periodic traveling wave solutions which are determined implicitly by (10) and $u(\xi)=\int_{\xi_{0}}^{\xi} v(\xi) d \xi$, where $\xi=k x+l y+m z+n t$ and $\xi_{0}$ is an arbitrary constant.

\section{3. $N$-Wave Solutions Linear Subspace of the $(3+1)$-Dimensional Jimbo-Miwa Equation}

In this section we study the $N$-wave solutions to the $(3+$ 1)-dimensional Jimbo-Miwa equation by the linear superposition principle to Hirota bilinear equations which was proposed firstly by Ma and Fan [30].

Let wave variables $\eta_{i}=\mathbf{k}_{\mathbf{i}} \cdot \mathbf{x}, 1 \leq i \leq N$, where $\mathbf{k}_{\mathbf{i}}=$ $\left\{k_{1, i}, k_{2, i}, k_{3, i}, k_{4, i}\right\}^{T}, \mathbf{x}=\{x, y, z, t\}^{T}$. According to the linear superposition principle to Hirota bilinear equations [30, 31], we can get the following sufficient and necessary conditions for $\operatorname{span}\left\{e^{\eta_{1}}, \ldots, e^{\eta_{N}}\right\}$ being a subspace of the Hirota bilinear equation (2).

Theorem 3. Let $\mathbf{k}_{\mathbf{i}}=\left\{k_{1, i}, k_{2, i}, k_{3, i}, k_{4, i}\right\}^{T}, \mathbf{x}=\{x, y, z, t\}^{T}$, and $f_{N}=\sum_{i=1}^{N} \epsilon_{i} e^{\mathbf{k}_{\mathbf{i}} \cdot \mathbf{x}}$. Then, for any constants $\left\{\epsilon_{i}, 1 \leq i \leq N\right\}, f_{N}$ solves the Hirota bilinear equation (2) if and only if

$$
\begin{gathered}
\left(k_{1, i}-k_{1, j}\right)^{3}\left(k_{2, i}-k_{2, j}\right)+2\left(k_{4, i}-k_{4, j}\right)\left(k_{2, i}-k_{2, j}\right) \\
-3\left(k_{1, i}-k_{1, j}\right)\left(k_{3, i}-k_{3, j}\right)=0
\end{gathered}
$$

for any $1 \leq j<i \leq N$.

It follows from Theorem 3 that $f_{N}$ is an $N$-wave solution to the Hirota bilinear equation (2) if (20) holds for any $1 \leq j<i \leq N$. However, the corresponding $u(x, y, z, t)=2\left(f_{N}\right)_{x} / f_{N}$ is not necessarily an $N$-wave solution to the $(3+1)$-dimensional Jimbo-Miwa equation (1) even if $\left\{\eta_{1}, \eta_{2}, \ldots, \eta_{N}\right\}$ is linear independent. In fact,

$$
\begin{aligned}
u(x, y, z, t) & =2 \frac{\left(f_{N}\right)_{x}}{f_{N}}=2 \frac{\sum_{i=1}^{N} \epsilon_{i} k_{1, i} e^{\mathbf{k}_{\mathbf{i}} \cdot \mathbf{x}}}{\sum_{i=1}^{N} \epsilon_{i} e^{\mathbf{k}_{\mathbf{i}} \cdot \mathbf{x}}} \\
& =2 \frac{k_{1,1}+\sum_{i=2}^{N} k_{1, i} \epsilon_{i}^{\prime} e^{\left(\mathbf{k}_{\mathbf{i}}-\mathbf{k}_{1}\right) \cdot \mathbf{x}}}{1+\sum_{i=2}^{N} \epsilon_{i}^{\prime} e^{\left(\mathbf{k}_{\mathbf{i}}-\mathbf{k}_{\mathbf{1}}\right) \cdot \mathbf{x}}} \\
& =2 k_{1,1}+2 \frac{\sum_{i=2}^{N} k_{1, i}^{\prime} \epsilon_{i}^{\prime} e^{\mathbf{k}_{\mathbf{i}}^{\prime} \cdot \mathbf{x}}}{1+\sum_{i=2}^{N} \epsilon_{i}^{\prime} e^{\mathbf{k}_{\mathbf{i}}^{\prime} \cdot \mathbf{x}}},
\end{aligned}
$$

where $\epsilon_{i}^{\prime}=\epsilon_{i} / \epsilon_{1}, k_{1, i}^{\prime}=k_{1, i}-k_{1,1}$ and $\mathbf{k}_{\mathbf{i}}^{\prime}=\mathbf{k}_{\mathbf{i}}-\mathbf{k}_{\mathbf{1}}$. Obviously, the function above is an $N-1$-wave solution to the $(3+$ 1)-dimensional Jimbo-Miwa equation (1) if $\left\{\eta_{i}^{\prime}, i=2,3, \ldots\right.$, $N\}$ is independent; that is, $\left\{\mathbf{k}_{\mathbf{i}}^{\prime}, i=2, \ldots, N\right\}$ is linear independent, solves (20), and satisfies the dispersion relations. Here, $\mathbf{k}_{\mathbf{i}}^{\prime}=\left\{k_{1, i}^{\prime}, k_{2, i}^{\prime}, k_{3, i}^{\prime}, k_{4, i}^{\prime}\right\}^{T}$ satisfies the dispersion relations meaning that $k_{1, i}^{\prime 3} k_{2, i}^{\prime}+2 k_{4, i}^{\prime} k_{2, i}^{\prime}-3 k_{1, i}^{\prime} k_{3, i}^{\prime}=0$ holds.

Recall that the goal of this paper is to investigate the multiwave solutions to the $(3+1)$-dimensional Jimbo-Miwa equation. Combining the conclusion of Theorem 3 with the analysis above, we can now state the following theorem on the $N$-wave solution to the $(3+1)$-dimensional Jimbo-Miwa equation (1).

Theorem 4. Let $\mathbf{k}_{\mathbf{i}}=\left\{k_{1, i}, k_{2, i}, k_{3, i}, k_{4, i}\right\}^{T}, \mathbf{x}=\{x, y, z, t\}^{T}$, $1 \leq i \leq N$. For any constants $g$ and $\left\{\epsilon_{i}, 1 \leq i \leq N\right\}$, suppose that

$$
u(x, y, z, t)=2 \frac{\sum_{i=1}^{N} k_{1, i} \epsilon_{i} e^{\mathbf{k}_{\mathbf{i}} \cdot \mathbf{x}}}{1+\sum_{i=1}^{N} \epsilon_{i} e^{\mathbf{k}_{\mathbf{i}} \cdot \mathbf{x}}}+g .
$$

Then $u(x, y, z, t)$ is an $N$-wave solution to the $(3+$ 1)-dimensional Jimbo-Miwa equation (1), if $\left\{\mathbf{k}_{\mathbf{1}}, \mathbf{k}_{\mathbf{2}}, \ldots, \mathbf{k}_{\mathbf{N}}\right\}$ is a linear independent solution set of equation (20) satisfying the dispersion relations.

To get other $N$-wave solutions to the $(3+1)$-dimensional Jimbo-Miwa equation, we study the linear independent solution sets to (20) which are required to satisfy the dispersion relations. Actually, (20) is a system possessing $N(N+1) / 2$ coupled equations (plus the dispersion conditions). It is usually not so easy to get the solutions of (20). Fortunately, the number of the equations is 1 when $N=1$ and it is 3 when $N=2$, which might make it easy to get the solutions in these two cases. Clearly, 1-wave solution is the traveling wave solution. Let us check what kinds of traveling wave solutions we can obtain by the Hirota bilinear method first.

For the case $N=1$ in (22), we only need to find the independent solutions $\mathbf{k}_{\mathbf{1}}$ of the dispersion relation

$$
\left(k_{1,1}\right)^{3} k_{2,1}+2 k_{4,1} k_{2,1}-3 k_{1,1} k_{3,1}=0 .
$$

That is to say, we get the traveling wave solutions to the $(3+1)$-dimensional Jimbo-Miwa equation from (20). Suppose that $l_{i}, i=1,2,3,4$ are any arbitrary constants, $l_{1} \neq 0$ and $l_{3}=$ $l_{2}\left(l_{1}^{3}+2 l_{4}\right) /\left(3 l_{1}\right)$; then $\mathbf{k}_{\mathbf{1}}=\left\{l_{1}, l_{2}, l_{3}, l_{4}\right\}^{T}$ satisfies (23), and thus

$$
u(x, y, z, t)=2 \frac{l_{1} \epsilon e^{\mathbf{k}_{\mathbf{i}} \cdot \mathbf{x}}}{1+\epsilon e^{\mathbf{k}_{\mathbf{i}} \cdot \mathbf{x}}}+g,
$$

where $\epsilon$ and $g$ are any arbitrary constants, is a 1-wave solution, that is, traveling wave solution to the $(3+1)$-dimensional Jimbo-Miwa equation.

By further computation, (24) could be rewritten as

$$
u(x, y, z, t)=g-\frac{2 l_{1}}{1+\epsilon e^{\mathbf{k}_{\mathbf{i}} \cdot \mathbf{x}}} .
$$

For $\epsilon>0$,

$$
\frac{2 l_{1}}{1+\epsilon e^{\mathbf{k}_{\mathbf{i}} \cdot \mathbf{x}}}=l_{1}\left[1-\tanh \frac{1}{2}\left(\mathbf{k}_{\mathbf{i}} \cdot \mathbf{x}+\xi_{\mathbf{0}}\right)\right]
$$

and, for $\epsilon<0$,

$$
\frac{2 l_{1}}{1+\epsilon e^{\mathbf{k}_{\mathbf{i}} \cdot \mathbf{x}}}=l_{1}\left[1-\operatorname{coth} \frac{1}{2}\left(\mathbf{k}_{\mathbf{i}} \cdot \mathbf{x}+\boldsymbol{\xi}_{\mathbf{0}}\right)\right],
$$

where $\xi_{0}=\ln |\epsilon|$ is an arbitrary constant. Consequently, (24) can be rewritten as

$$
\begin{aligned}
& u(x, y, z, t)=g-l_{1} \tanh \frac{1}{2}\left(\mathbf{k}_{\mathbf{i}} \cdot \mathbf{x}+\xi_{\mathbf{0}}\right), \\
& u(x, y, z, t)=g-l_{1} \operatorname{coth} \frac{1}{2}\left(\mathbf{k}_{\mathbf{i}} \cdot \mathbf{x}+\boldsymbol{\xi}_{\mathbf{0}}\right) .
\end{aligned}
$$


By parametric transformation, we can find that the traveling wave solution (28) is exactly the same as solution (19) in Section 2. However, (29) is a family of unbounded solutions.

3.1. 2-Wave Solutions to the $(3+1)$-Dimensional Jimbo-Miwa Equation. To obtain the 2 -wave solutions to the $3+$ 1)-dimensional Jimbo-Miwa equation, we now study the solutions to the following coupled algebraic equations:

$$
\begin{gathered}
k_{1,1}^{3} k_{2,1}+2 k_{4,1} k_{2,1}-3 k_{1,1} k_{3,1}=0, \\
k_{1,2}^{3} k_{2,2}+2 k_{4,2} k_{2,2}-3 k_{1,2} k_{3,2}=0 \\
\left(k_{1,2}-k_{1,1}\right)^{3}\left(k_{2,2}-k_{2,1}\right)+2\left(k_{4,2}-k_{4,1}\right)\left(k_{2,2}-k_{2,1}\right) \\
-3\left(k_{1,2}-k_{1,1}\right)\left(k_{3,2}-k_{3,1}\right)=0 .
\end{gathered}
$$

From (30), we get

$$
\begin{aligned}
k_{3,1} & =\frac{k_{1,1}^{3}+2 k_{4,1}}{3 k_{1,1}} k_{2,1}, \\
k_{3,2} & =\frac{k_{1,2}^{3}+2 k_{4,2}}{3 k_{1,2}} k_{2,2}, \\
k_{3,2}-k_{3,1}= & \frac{\left(k_{1,2}-k_{1,1}\right)^{3}+2\left(k_{4,2}-k_{4,1}\right)}{3\left(k_{1,2}-k_{1,1}\right)} \\
& \times\left(k_{2,2}-k_{2,1}\right) .
\end{aligned}
$$

Clearly, from (31)-(33), we have

$$
\begin{gathered}
\frac{\left(k_{1,2}-k_{1,1}\right)^{3}+2\left(k_{4,2}-k_{4,1}\right)}{3\left(k_{1,2}-k_{1,1}\right)}\left(k_{2,2}-k_{2,1}\right) \\
=\frac{k_{1,2}^{3}+2 k_{4,2}}{3 k_{1,2}} k_{2,2}-\frac{k_{1,1}^{3}+2 k_{4,1}}{3 k_{1,1}} k_{2,1},
\end{gathered}
$$

from which we get

$$
\begin{aligned}
k_{2,2}=( & \left(\left(k_{1,1}^{3}+2 k_{4,1}\right)\left(k_{1,2}-k_{1,1}\right)\right. \\
& \left.-k_{1,1}\left[\left(k_{1,2}-k_{1,1}\right)^{3}+2\left(k_{4,2}-k_{4,1}\right)\right]\right) \\
& \times\left(\left(k_{1,2}^{3}+2 k_{4,2}\right)\left(k_{1,2}-k_{1,1}\right)\right. \\
& \left.\left.-k_{1,2}\left[\left(k_{1,2}-k_{1,1}\right)^{3}+2\left(k_{4,2}-k_{4,1}\right)\right]\right)^{-1}\right) \\
\times & \frac{k_{1,1}}{k_{1,2}} k_{2,1} .
\end{aligned}
$$

Now, substituting (35) into (32), we obtain

$$
\begin{aligned}
k_{3,2}=( & \left(\left(k_{1,1}^{3}+2 k_{4,1}\right)\left(k_{1,2}-k_{1,1}\right)\right. \\
& \left.-k_{1,1}\left[\left(k_{1,2}-k_{1,1}\right)^{3}+2\left(k_{4,2}-k_{4,1}\right)\right]\right) \\
& \times\left(\left(k_{1,2}^{3}+2 k_{4,2}\right)\left(k_{1,2}-k_{1,1}\right)\right.
\end{aligned}
$$

$$
\begin{aligned}
& \left.\left.-k_{1,2}\left[\left(k_{1,2}-k_{1,1}\right)^{3}+2\left(k_{4,2}-k_{4,1}\right)\right]\right)^{-1}\right) \\
& \times \frac{\left(k_{1,2}^{3}+2 k_{4,2}\right)}{3 k_{1,2}^{2}} k_{1,1} k_{2,1} .
\end{aligned}
$$

Consequently, we get the 2 -wave solutions to the $(3+$ 1)-dimensional Jimbo-Miwa equation and we can now state the following theorem.

Theorem 5. Let $\mathbf{k}_{\mathbf{i}}=\left\{k_{1, i}, k_{2, i}, k_{3, i}, k_{4, i}\right\}^{T}, \mathbf{x}=\{x, y, z, t\}^{T}, i=$ 1,2 . For any constants $g$ and $\left\{\epsilon_{i}, i=1,2\right\}$,

$$
u(x, y, z, t)=2 \frac{k_{1,1} \epsilon_{1} e^{\mathbf{k}_{1} \cdot \mathbf{x}}+k_{1,2} \epsilon_{2} e^{\mathbf{k}_{2} \cdot \mathbf{x}}}{1+\epsilon_{1} e^{\mathbf{k}_{1} \cdot \mathbf{x}}+\epsilon_{2} e^{\mathbf{k}_{2} \cdot \mathbf{x}}}+g
$$

is a 2-wave solution to the $(3+1)$-dimensional Jimbo-Miwa equation (1), where $k_{1,1}, k_{1,2}, k_{2,1}, k_{4,1}$, and $k_{4,2}$ are arbitrary constants and the constants $k_{3,1}, k_{2,2}$, and $k_{3,2}$ are determined by (31), (35), and (36), respectively.

3.2. N-Wave Solutions to the $(3+1)$-Dimensional JimboMiwa Equation. To get the $N$-wave solutions to the $(3+$ 1)-dimensional Jimbo-Miwa equation, we need to investigate the independent solution set $\left\{\mathbf{k}_{\mathbf{1}}, \mathbf{k}_{\mathbf{2}}, \ldots, \mathbf{k}_{\mathrm{N}}\right\}$ of (20), which satisfies the dispersion relations. Generally, it is very difficult to get the solution if $N$ is greater than 3 in which case the number of (20) is $N(N+1) / 2$. However, it might be possible to solve these equations by assuming some special relations between these parameters [30, 31].

Following the idea in $[30,31]$, suppose that $\mathbf{k}_{\mathbf{i}}=$ $\left\{a_{1} k_{i}^{n_{1}}, a_{2} k_{i}^{n_{2}}, a_{3} k_{i}^{n_{3}}, a_{4} k_{i}^{n_{4}}\right\}^{T}, \quad i=1,2, \ldots, N$, where $a_{j}^{\prime} s$ and $n_{j}^{\prime} s, j=1,2,3,4$, are parameters to be determined later. To get the possible solutions to (20), we choose $\left(n_{1}, n_{2}, n_{3}, n_{4}\right)$ to be $(1,2,4,3)$ or $(1,-1,1,3)$.

For the case when $\left(n_{1}, n_{2}, n_{3}, n_{4}\right)=(1,2,4,3)$, substituting $\mathbf{k}_{\mathbf{i}}=\left\{a_{1} k_{i}^{n_{1}}, a_{2} k_{i}^{n_{2}}, a_{3} k_{i}^{n_{3}}, a_{4} k_{i}^{n_{4}}\right\}^{T}, i=1,2, \ldots, N$, into (20) gives

$$
\begin{gathered}
a_{1}^{3} a_{2}\left(k_{i}-k_{j}\right)^{3}\left(k_{i}^{2}-k_{j}^{2}\right)+2 a_{4} a_{2}\left(k_{i}^{3}-k_{j}^{3}\right)\left(k_{i}^{2}-k_{j}^{2}\right) \\
-3 a_{1} a_{4}\left(k_{i}-k_{j}\right)\left(k_{i}^{4}-k_{j}^{4}\right)=0 .
\end{gathered}
$$

Obviously, (38) holds for arbitrary values of $k_{i}$ and $k_{j}$ if and only if $a_{1}, a_{2}, a_{3}$, and $a_{4}$ satisfy the two equations

$$
\begin{gathered}
2 a_{2}\left(a_{1}^{3}+2 a_{4}\right)-3 a_{1} a_{3}=0, \\
a_{2}\left(a_{1}^{3}-4 a_{4}\right)=0 .
\end{gathered}
$$

Solving (39), we get $a_{4}=(1 / 4) a_{1}^{3}, a_{3}=a_{1}^{2} a_{2}$, where $a_{1}$ and $a_{2}$ are free parameters. It is easy to see that $\mathbf{k}_{\mathbf{i}}=$ $\left\{a_{1} k_{i}, a_{2} k_{i}^{2}, a_{3} k_{i}^{4}, a_{4} k_{i}^{3}\right\}^{T}, i=1,2, \ldots, N$, satisfy the dispersion condition. Consequently, we know that the $(3+$ 1)-dimensional Jimbo-Miwa equation (1) possesses $N$-wave solution for any arbitrary positive integer $N$. Thus, we have the following theorem. 
Theorem 6. For any arbitrary positive integer $N$ and for any different $k_{i}, i=1,2, \ldots, N$,

$$
\begin{aligned}
& u(x, y, z, t) \\
& \quad=2 a_{1} \frac{\sum_{i=1}^{N} k_{i} \epsilon_{i} e^{a_{1} k_{i} x+a_{2} k_{i}^{2} y+a_{1}^{2} a_{2} k_{i}^{4} z+(1 / 4) a_{1}^{3} k_{i}^{3} t}}{1+\sum_{i=1}^{N} \epsilon_{i} e^{a_{1} k_{i} x+a_{2} k_{i}^{2} y+a_{1}^{2} a_{2} k_{i}^{4} z+(1 / 4) a_{1}^{3} k_{i}^{3} t}}+g
\end{aligned}
$$

is a family of $N$-wave solutions to the $(3+1)$-dimensional Jimbo-Miwa equation (1), where $\epsilon_{i}, i=1,2, \ldots, N$, and $a_{1}$ and $a_{2}$ are arbitrary constants.

For the case when $\left(n_{1}, n_{2}, n_{3}, n_{4}\right)=(1,-1,1,3)$, substituting $\mathbf{k}_{\mathbf{i}}=\left\{a_{1} k_{i}, a_{2} k_{i}^{-1}, a_{3} k_{i}, a_{4} k_{i}^{3}\right\}^{T}, i=1,2, \ldots, N$, into (20) gives

$$
\begin{gathered}
a_{1}^{3} a_{2}\left(k_{i}-k_{j}\right)^{3}\left(k_{i}^{-1}-k_{j}^{-1}\right)+2 a_{4} a_{2}\left(k_{i}^{3}-k_{j}^{3}\right)\left(k_{i}^{-1}-k_{j}^{-1}\right) \\
-3 a_{1} a_{4}\left(k_{i}-k_{j}\right)\left(k_{i}^{3}-k_{j}^{3}\right)=0 .
\end{gathered}
$$

Clearly, (41) holds for arbitrary values of $k_{i}$ and $k_{j}$ if and only if $a_{1}, a_{2}, a_{3}$, and $a_{4}$ satisfy

$$
\begin{gathered}
2 a_{2}\left(a_{1}^{3}-a_{4}\right)-3 a_{1} a_{3}=0, \\
a_{2}\left(a_{1}^{3}+2 a_{4}\right)=0 .
\end{gathered}
$$

Solving (42), we obtain $a_{4}=-a_{1}^{3} / 2, a_{3}=a_{1}^{2} a_{2}$, where $a_{1}$ and $a_{2}$ are free parameters. Unfortunately, unlike the case when $\left(n_{1}, n_{2}, n_{3}, n_{4}\right)=(1,2,4,3)$ above, it is easy to see that $\mathbf{k}_{\mathbf{i}}=\left\{a_{1} k_{i}, a_{2} k_{i}^{-1}, a_{3} k_{i}, a_{4} k_{i}^{3}\right\}^{T}, i=1,2, \ldots, N$, do not satisfy the dispersion condition any more. However, if we let $\mathbf{k}_{\mathbf{0}}=$ $\left\{a_{1}, a_{2}, a_{3}, a_{4}\right\}^{T}$, then, for any $i=1,2, \ldots, N$,

$$
\begin{array}{r}
\mathbf{k}_{i}^{\prime}=\mathbf{k}_{i}-\mathbf{k}_{\mathbf{0}}=\left\{a_{1}\left(k_{i}-1\right), a_{2}\left(k_{i}^{-1}-1\right),\right. \\
\left.a_{3}\left(k_{i}-1\right), a_{4}\left(k_{i}^{3}-1\right)\right\}^{T}
\end{array}
$$

satisfies the dispersion relations because $\left\{\mathbf{k}_{\mathbf{0}}, \mathbf{k}_{\mathbf{1}}, \mathbf{k}_{\mathbf{2}}, \ldots, \mathbf{k}_{\mathbf{N}}\right\}$ is a solution set of (20).

Consequently, besides the family of $N$-wave solution (40), the $(3+1)$-dimensional Jimbo-Miwa equation (1) has another group of $N$-wave solution for any arbitrary positive integer $N$ and so we have the following theorem.

Theorem 7. For any arbitrary positive integer $N$ and for any different $k_{i}, i=1,2, \ldots, N$,

$$
\begin{aligned}
& u(x, y, z, t) \\
& =2 a_{1} \frac{\sum_{i=1}^{N}\left(k_{i}-1\right) \epsilon_{i} e^{a_{1}\left(k_{i}-1\right) x+a_{2}\left(k_{i}^{-1}-1\right) y+a_{1}^{2} a_{2}\left(k_{i}-1\right) z-(1 / 2) a_{1}^{3}\left(k_{i}^{3}-1\right) t}}{1+\sum_{i=1}^{N} \epsilon_{i} e^{a_{1}\left(k_{i}-1\right) x+a_{2}\left(k_{i}^{-1}-1\right) y+a_{1}^{2} a_{2}\left(k_{i}-1\right) z-(1 / 2) a_{1}^{3}\left(k_{i}^{3}-1\right) t}}
\end{aligned}
$$$$
+g
$$

is a family of $N$-wave solutions to the $(3+1)$-dimensional Jimbo-Miwa equation (1), where $\epsilon_{i}, i=1,2, \ldots, N$, and $a_{1}$ and $a_{2}$ are arbitrary constants.

\section{Concluding Remarks}

The dynamical system theory was employed to study the traveling wave solutions of the $(3+1)$-dimensional JimboMiwa equation (1). The multiwave solutions were investigated by studying the linear space of the corresponding Hirota bilinear equation. The exact formulas of two families of multiwave solutions of any order were obtained as well. Wazwaz [38] employed Hirota's bilinear method and derived 1-wave and 2-wave solutions to this equation and stated that it is completely integrable and it admits multiple-soliton solutions of any order. In this paper we explicitly obtained two different families of $N$-wave solutions to the $(3+1)$-dimensional Jimbo-Miwa equation (1) given by (40) and (44) and this confirmed the statement given in [38] that the Jimbo-Miwa equation (1) admits multiple-wave solutions of any order $N$.

\section{Conflict of Interests}

The authors declare that there is no conflict of interests regarding the publication of this paper.

\section{Acknowledgments}

This work is supported by the Nature Science Foundation of China (no. 11101371). This paper is motivated by nice discussion with Professor Wen-Xiu Ma during the authors' visit at University of South Florida in March 2013. The authors would like to express their sincere appreciations to Professor $\mathrm{Ma}$ and the anonymous reviewers.

\section{References}

[1] J.-H. He and X.-H. Wu, "Exp-function method for nonlinear wave equations," Chaos, Solitons \& Fractals, vol. 30, no. 3, pp. 700-708, 2006.

[2] E. J. Parkes and B. R. Duffy, "An automated tanh-function method for finding solitary wave solutions to non-linear evolution equations," Computer Physics Communications, vol. 98, no. 3, pp. 288-300, 1996.

[3] M. Wang, Y. Zhou, and Z. Li, "Application of a homogeneous balance method to exact solutions of nonlinear equations in mathematical physics," Physics Letters A, vol. 216, no. 1-5, pp. $67-75,1996$.

[4] Z. Feng, "Comment on 'on the extended applications of homogeneous balance method"' Applied Mathematics and Computation, vol. 158, no. 2, pp. 593-596, 2004.

[5] S. Zhang and T. Xia, "A generalized new auxiliary equation method and its applications to nonlinear partial differential equations," Physics Letters A, vol. 363, no. 5-6, pp. 356-360, 2007.

[6] H. Zhang, "New exact travelling wave solutions of nonlinear evolution equation using a sub-equation," Chaos, Solitons \& Fractals, vol. 39, no. 2, pp. 873-881, 2009.

[7] W. X. Ma, "Travelling wave solutions to a seventh order generalized KdV equation," Physics Letters A, vol. 180, no. 3, pp. 221224, 1993.

[8] C. Yan, "A simple transformation for nonlinear waves," Physics Letters A, vol. 224, no. 1-2, pp. 77-84, 1996. 
[9] A.-M. Wazwaz, "A sine-cosine method for handling nonlinear wave equations," Mathematical and Computer Modelling, vol. 40, no. 5-6, pp. 499-508, 2004.

[10] H. B. Lan and K. L. Wang, "Exact solutions for two nonlinear equations. I," Journal of Physics A. Mathematical and General, vol. 23, no. 17, pp. 3923-3928, 1990.

[11] E. J. Parkes, B. R. Duffy, and P. C. Abbott, "The Jacobi elliptic-function method for finding periodic-wave solutions to nonlinear evolution equations," Physics Letters A, vol. 295, no. 5-6, pp. 280-286, 2002.

[12] G. L. Cai, Q. C. Wang, and J. J. Huang, "A modified F-expansion method for solving breaking soliton equation," International Journal of Nonlinear Science, vol. 2, pp. 122-128, 2006.

[13] W. Hereman and W. Zhuang, "Symbolic computation of solitons with Macsyma," in Computational and Applied Mathematics, II (Dublin, 1991), pp. 287-296, North-Holland, Amsterdam, The Netherlands, 1992.

[14] N. A. Kudryashov, "Seven common errors in finding exact solutions of nonlinear differential equations," Communications in Nonlinear Science and Numerical Simulation, vol. 14, no. 9-10, pp. 3507-3529, 2009.

[15] L. Zhang and X. Huo, "On the exp-function method for constructing travelling wave solutions of nonlinear equations," in Nonlinear and Modern Mathematical Physics: Proceedings of the First International Workshop, vol. 1212, pp. 280-285, American Institute of Physics, Melville, NY, USA, 2010.

[16] J. B. Li, Singular Traveling Wave Equations: Bifurcations and Exact Solutions, Science Press, Beijing, China, 2013.

[17] L. Zhang, L.-Q. Chen, and X. Huo, "The effects of horizontal singular straight line in a generalized nonlinear Klein-Gordon model equation," Nonlinear Dynamics, vol. 72, no. 4, pp. 789801, 2013.

[18] R. Hirota, The Direct Method in Soliton Theory, vol. 155 of Cambridge Tracts in Mathematics, Cambridge University Press, Cambridge, Mass, USA, 2004.

[19] J. Hietarinta, "Hirota's bilinear method and soliton solutions," Physics AUC, vol. 15, part 1, pp. 31-37, 2005.

[20] Y. Ye, L. Wang, Z. Chang, and J. He, "An efficient algorithm of logarithmic transformation to Hirota bilinear form of KdV-type bilinear equation," Applied Mathematics and Computation, vol. 218, no. 5, pp. 2200-2209, 2011.

[21] Z. Zhou, J. Fu, and Z. Li, "An implementation for the algorithm of Hirota bilinear form of PDE in the Maple system," Applied Mathematics and Computation, vol. 183, no. 2, pp. 872-877, 2006.

[22] Z. Zhou, J. Fu, and Z. Li, "Maple packages for computing Hirota's bilinear equation and multisoliton solutions of nonlinear evolution equations," Applied Mathematics and Computation, vol. 217, no. 1, pp. 92-104, 2010.

[23] X.-D. Yang and H.-Y. Ruan, "A Maple package on symbolic computation of Hirota bilinear form for nonlinear equations," Communications in Theoretical Physics, vol. 52, no. 5, pp. 801-807, 2009.

[24] J. Hietarinta, "A search for bilinear equations passing Hirota's three-soliton condition. II. mKdV-type bilinear equations," Journal of Mathematical Physics, vol. 28, no. 9, pp. 2094-2101, 1987.

[25] J. Hietarinta, "A search for bilinear equations passing Hirota's three-soliton condition. III. Sine-Gordon-type bilinear equations," Journal of Mathematical Physics, vol. 28, no. 11, pp. 25862592, 1987.
[26] J. Hietarinta, "A search for bilinear equations passing Hirota's three-soliton condition. IV. Complex bilinear equations," Journal of Mathematical Physics, vol. 29, no. 3, pp. 628-635, 1988.

[27] W.-X. Ma, T. Huang, and Y. Zhang, "A multiple exp-function method for nonlinear differential equations and its application," Physica Scripta, vol. 82, no. 6, Article ID 065003, 2010.

[28] W.-X. Ma and W. Strampp, "Bilinear forms and Bäcklund transformations of the perturbation systems," Physics Letters A, vol. 341, no. 5-6, pp. 441-449, 2005.

[29] W.-X. Ma, R. Zhou, and L. Gao, "Exact one-periodic and twoperiodic wave solutions to Hirota bilinear equations in $(2+1)$ dimensions," Modern Physics Letters A, vol. 24, no. 21, pp. 1677$1688,2009$.

[30] W.-X. Ma and E. Fan, "Linear superposition principle applying to Hirota bilinear equations," Computers \& Mathematics with Applications, vol. 61, no. 4, pp. 950-959, 2011.

[31] W.-X. Ma, Y. Zhang, Y. Tang, and J. Tu, "Hirota bilinear equations with linear subspaces of solutions," Applied Mathematics and Computation, vol. 218, no. 13, pp. 7174-7183, 2012.

[32] W. X. Ma, "Generalized bilinear differential equations," Studies in Nonlinear Sciences, vol. 2, no. 4, pp. 140-144, 2011.

[33] M. Jimbo and T. Miwa, "Solitons and infinite-dimensional Lie algebras," Publications of the Research Institute for Mathematical Sciences, vol. 19, no. 3, pp. 943-1001, 1983.

[34] W.-X. Ma and J.-H. Lee, "A transformed rational function method and exact solutions to the $3+1$ dimensional JimboMiwa equation," Chaos, Solitons \& Fractals, vol. 42, no. 3, pp. 1356-1363, 2009.

[35] Z. Li and Z. Dai, "Abundant new exact solutions for the $(3+$ 1)-dimensional Jimbo-Miwa equation," Journal of Mathematical Analysis and Applications, vol. 361, no. 2, pp. 587-590, 2010.

[36] N. A. Kudryashov and D. I. Sinelshchikov, "A note on "Abundant new exact solutions for the $(3+1)$-dimensional Jimbo-Miwa equation," Journal of Mathematical Analysis and Applications, vol. 371, no. 1, pp. 393-396, 2010.

[37] S. N. Chow and J. K. Hale, Methods of Bifurcation Theory, Springer, New York, NY, USA, 1981.

[38] A.-M. Wazwaz, "Multiple-soliton solutions for the CalogeroBogoyavlenskii-Schiff, Jimbo-Miwa and YTSF equations," Applied Mathematics and Computation, vol. 203, no. 2, pp. 592-597, 2008. 


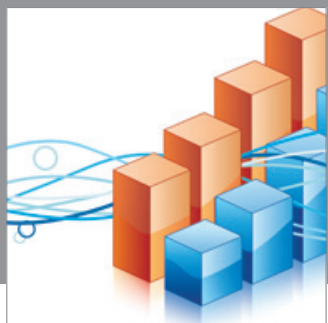

Advances in

Operations Research

mansans

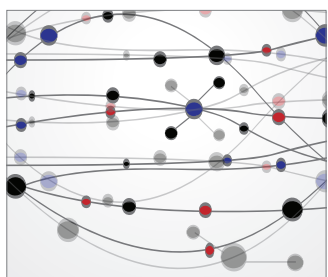

The Scientific World Journal
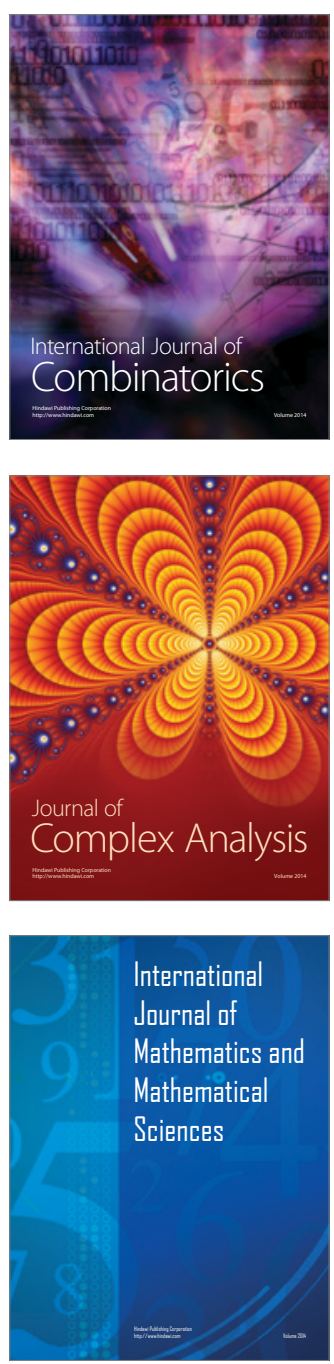
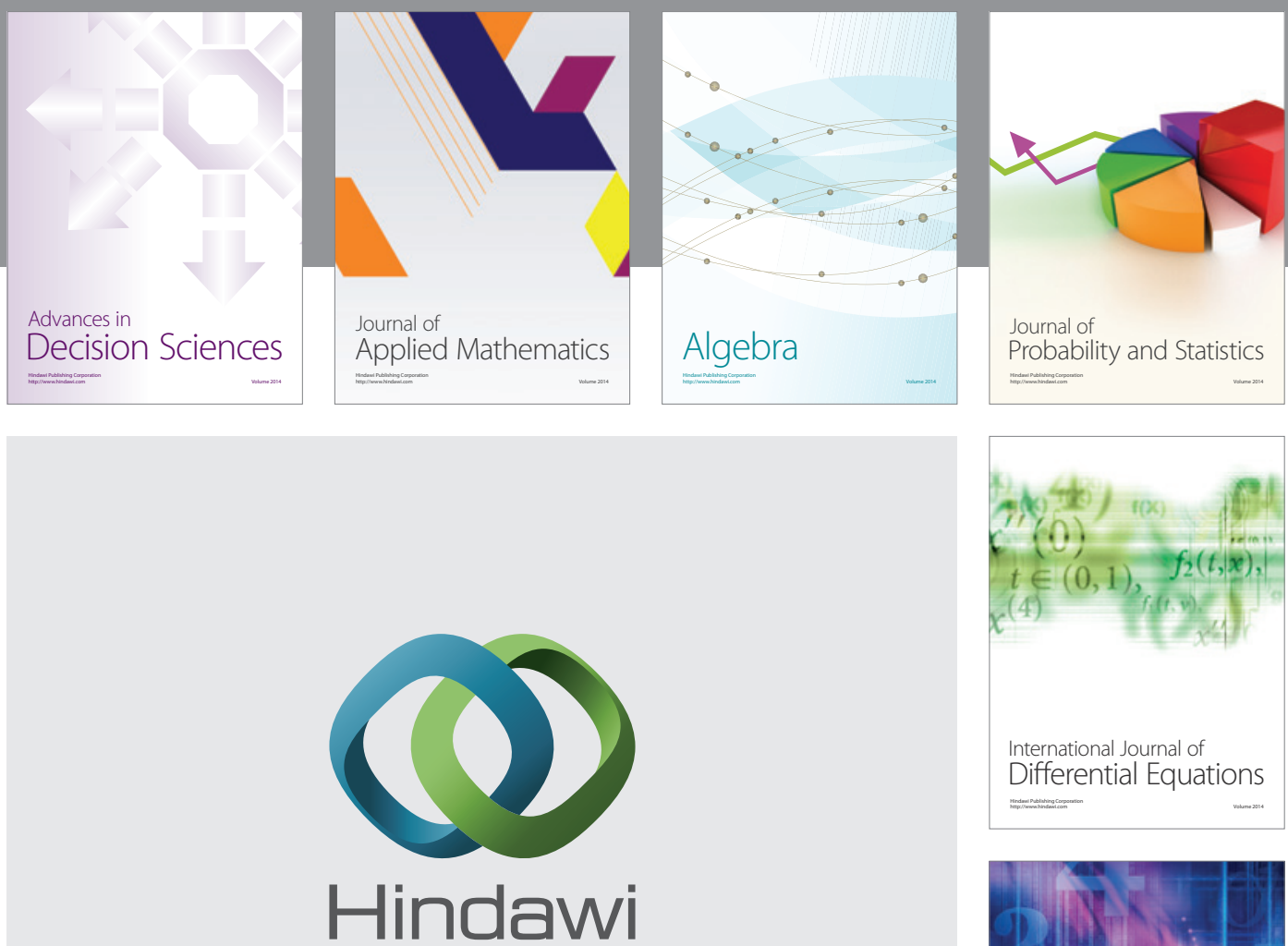

Submit your manuscripts at http://www.hindawi.com
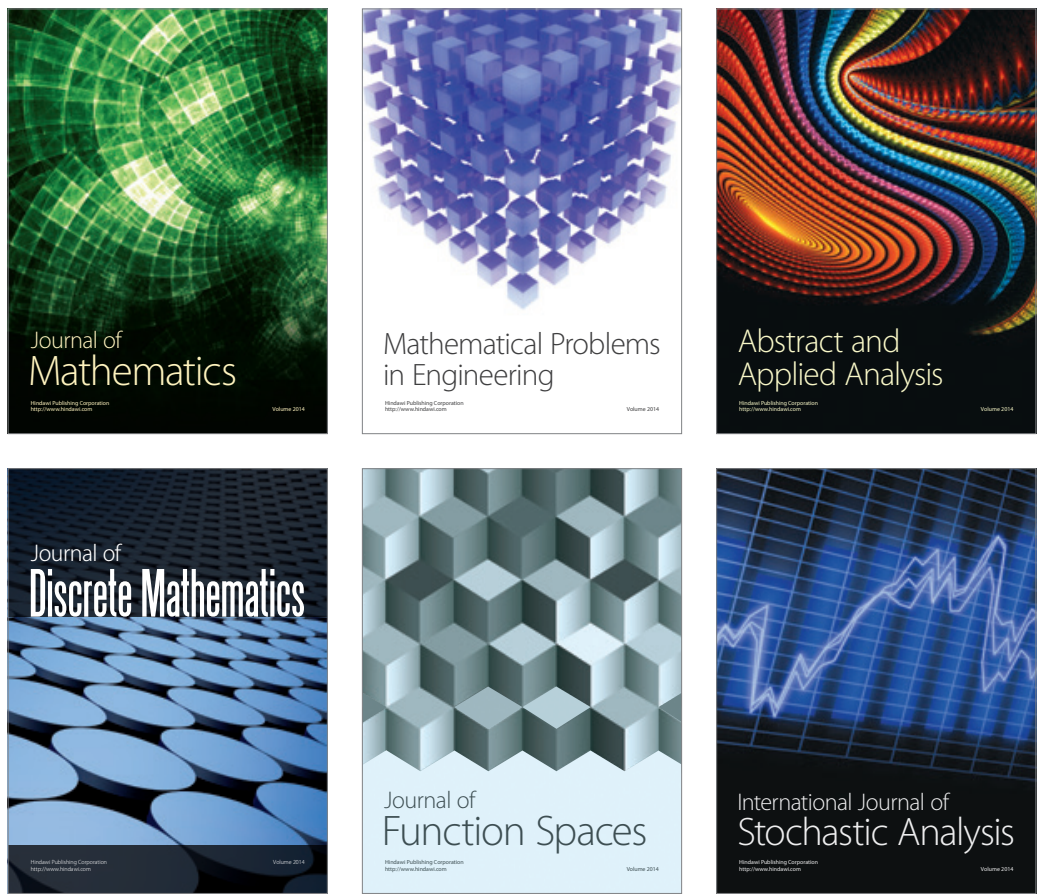

Journal of

Function Spaces

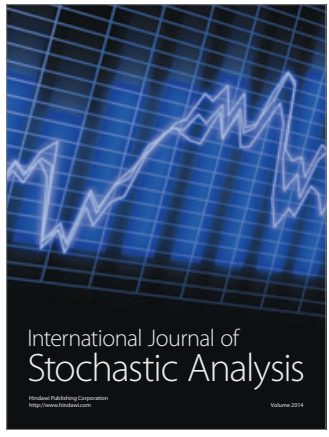

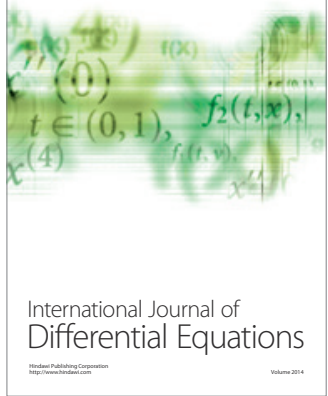
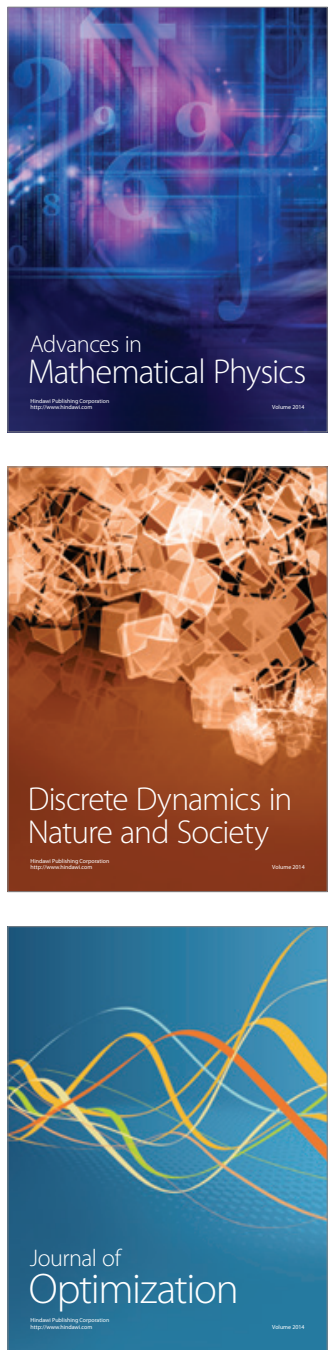